\title{
OPTIMIZATION PROBLEM FOR EXTREMALS OF THE TRACE INEQUALITY IN DOMAINS WITH HOLES
}

\author{
LEANDRO M. DEL PEZZO
}

\begin{abstract}
We study the Sobolev trace constant for functions defined in a bounded domain $\Omega$ that vanish in the subset $A$. We find a formula for the first variation of the Sobolev trace with respect to hole. As a consequence of this formula, we prove that when $\Omega$ is a centered ball, the symmetric hole is critical when we consider deformation that preserve volume but is not optimal for some case.
\end{abstract}

\section{Introduction and Main Results.}

Let $\Omega$ be a bounded smooth domain in $\mathbb{R}^{N}$ with $N \geq 2$ and $1<p<\infty$. We denote by $p_{*}$ the critical exponent for the Sobolev trace immersion given by $p_{*}=p(N-1) /(N-p)$ if $p<N$ and $p_{*}=\infty$ if $p \geq N$.

For any $A \subset \bar{\Omega}$, which is a smooth open subset, we define the space

$$
W_{A}^{1, p}(\Omega)=\overline{C_{0}^{\infty}(\bar{\Omega} \backslash A)},
$$

where the closure is taken in $W^{1, p}$-norm. By the Sobolev Trace Theorem, there is a compact embedding

$$
W_{A}^{1, p}(\Omega) \hookrightarrow L^{q}(\partial \Omega),
$$

for all $1<q<p^{*}$. Thus, given $1<q<p^{*}$, there exist a constant $C=C(q, p)$ such that

$$
C\left\{\int_{\partial \Omega}|u|^{q} \mathrm{~d} S\right\}^{\frac{p}{q}} \leq \int_{\Omega}|\nabla u|^{p}+|u|^{p} \mathrm{~d} x .
$$

The best (largest) constant in the above inequality is given by

$$
S_{q}(A):=\inf _{u \in W_{A}^{1, p}(\Omega) \backslash W_{0}^{1, p}(\Omega)} \frac{\int_{\Omega}|\nabla u|^{p}+|u|^{p} \mathrm{~d} x}{\left\{\int_{\partial \Omega}|u|^{q} \mathrm{~d} S\right\}^{\frac{p}{q}}} .
$$

By (1.1), there exist an extremal for $S_{q}(A)$. Moreover, an extremal for $S_{q}(A)$ is a weak solution to

$$
\begin{cases}-\Delta_{p} u+|u|^{p-2} u=0 & \text { in } \Omega \backslash \bar{A} \\ |\nabla u|^{p-2} \frac{\partial u}{\partial \nu}=\lambda|u|^{q-2} u & \text { on } \partial \Omega, \\ u=0 & \text { on } \partial A\end{cases}
$$

where $\Delta_{p} u=\operatorname{div}\left(|\nabla u|^{p-2} \nabla u\right)$ is the usual $p$-laplacian, $\frac{\partial}{\partial \nu}$ is the outer unit normal derivative and $\lambda$ depends on the normalization of $u$. When $\|u\|_{L^{q}(\partial \Omega)}=1$ we have that $\lambda=S_{q}(A)$. Moreover, when $p=q$ problem (1.3) becomes homogeneous and therefore is a nonlinear eigenvalue problem. In this case, the first eigenvalue of (1.3) coincides with the best Sobolev trace constant $S_{q}(A)=\lambda_{1}(A)$ and it is shown 
in [9] that it is simple (see also [3]). Therefore, if $p=q$, the extremal for $S_{p}(A)$ is unique up to constant factor. In the linear setting, i.e. when $p=q=2$, this eigenvalue problem is known as the Steklov eigenvalue problem, see [10].

It is the purpose of this article to analyze the dependance of the Sobolev trace constant $S_{q}(A)$ with respect to variations on the set $A$. To this end, we compute the so-called shape derivative of $S_{q}(A)$ with respect to regular perturbations of the hole A.

Let $V: \mathbb{R}^{N} \rightarrow \mathbb{R}^{N}$ be a regular (smooth) vector filed, globally Lipschitz, with support in $\Omega$ and let $\psi_{t}: \mathbb{R}^{N} \rightarrow \mathbb{R}^{N}$ be defined as the unique solution to

$$
\begin{cases}\frac{d}{d t} \psi_{t}(x)=V\left(\psi_{t}(x)\right) & t>0 \\ \psi_{0}(x)=x & x \in \mathbb{R}^{N} .\end{cases}
$$

We have

$$
\psi_{t}(x)=x+t V(x)+o(t) \quad \forall x \in \mathbb{R}^{N} .
$$

Now, we define $A_{t}:=\psi_{t}(A) \subset \Omega$ for all $t>0$ and

$$
S_{q}(t)=\inf _{u \in W_{A_{t}}^{1, p}(\Omega) \backslash W_{0}^{1, p}(\Omega)} \frac{\int_{\Omega}|\nabla u|^{p}+|u|^{p} \mathrm{~d} x}{\left\{\int_{\partial \Omega}|u|^{q} \mathrm{~d} S\right\}^{\frac{p}{q}}} .
$$

Observe that $A_{0}=A$ and therefore $S_{q}(0)=S_{q}(A)$.

In [2] Fernández Bonder, Groisman and Rossi analyze this problem in the linear case $p=q=2$ and prove that $S_{2}(t)$ is differentiable with respect to $t$ at $t=0$ and it holds

$$
\left.\frac{d}{d t} S_{2}(t)\right|_{t=0}=-\int_{\partial A}\left(\frac{\partial u}{\partial \nu}\right)^{2}\langle V, \nu\rangle \mathrm{d} S,
$$

where $u$ is a normalized eigenfunction for $S_{2}(A)$ and $\nu$ is the exterior normal vector to $\Omega \backslash \bar{A}$.

Furthermore, in the case that $\Omega$ is the ball $B_{R}$ with center 0 and radius $R>0$ the authors show that a centered ball $A=B_{r}, r<R$, is critical in the sense that $S_{2}^{\prime}(A)=0$ when considering deformations that preserves volume and that this configuration is not optimal.

We say that hole $A^{*}$ is optimal for the parameter $\alpha, 0<\alpha<|\Omega|$, if $\left|A^{*}\right|=\alpha$ and

$$
S_{q}\left(A^{*}\right)=\inf _{\substack{A \subset \bar{\Omega} \\|A|=\alpha}} S_{q}(A) .
$$

Therefore there is a lack of symmetry in the optimal configuration.

Here we extend these results to the more general case $1<p<\infty$ and $1<q<p^{*}$. Our method differs from the one in [2] in order to deal with the nonlinear character of the problem.

Our first result states

Theorem 1.1. Suppose $A \subset \bar{\Omega}$ is a smooth open subset and let $1<q<p^{*}$. Then, with the previous notation, we have that $S_{q}(t)$ is differentiable at $t=0$ and there exists $u$ a normalized extremal for $S_{q}(A)$ such that

$$
S_{q}^{\prime}(0)=-\int_{\partial A}\left|\frac{\partial u}{\partial \nu}\right|^{p}\langle V, \nu\rangle \mathrm{d} S
$$

where $S_{q}^{\prime}(0)=\left.\frac{d}{d t} S_{q}(t)\right|_{t=0}$ and $\nu$ is the exterior normal vector to $\Omega \backslash \bar{A}$. 
Remark 1.2. If $u$ is an extremal for $S_{q}(A)$ we have that $|u|$ is also an extremal associated to $S_{q}(A)$. Then in the previous theorem we can suppose that $u \geq 0$ in $\Omega$. Moreover, by [8], we have that $u \in C^{1, \alpha}(\bar{\Omega})$ and if $\Omega$ satisfies the interior ball condition for all $x \in \partial \Omega$ then $u>0$ on $\partial \Omega$, see [11].

In the case that $\Omega=B_{R}$, we have the next result

Theorem 1.3. Let $\Omega=B_{R}$ and let the hole be a centered ball $A=B_{r}$. Then, if $1<q \leq p$, this configuration is critical in the sense that $S_{q}^{\prime}\left(B_{r}\right)=0$ for all deformations $V$ that preserve the volume of $B_{r}$.

But, if $q$ is sufficiently large, the symmetric hole with a radial extremal is not an optimal configuration. In fact, we prove

Theorem 1.4. Let $r>0$ and $1<p<\infty$ be fixed. Let $R>r$ and

$$
Q(R)=\frac{1}{S_{p}\left(B_{r}\right)^{\frac{p}{p-1}}}\left(1-\frac{N-1}{R} S_{p}\left(B_{r}\right)\right)+1 .
$$

If $q>Q(R)$ then the centered hole $B_{r}$ is not optimal.

Finally, to study the asymptotic behavior of $Q(R)$

Proposition 1.5. The function $Q(R)$ has the following asymptotic behavior

$$
\lim _{R \rightarrow r} Q(R)=1^{-} \quad \text { and } \lim _{R \rightarrow+\infty} Q(R)=p
$$

Observe that $Q(R)<1$ for $R$ close to $r$ and therefore the symmetric hole with a radial extremal is not an optimal configuration for $R$ close to $r$.

\section{Proof of Theorem 1.1}

2.1. Preliminary Results. The proof of Theorem 1.1 require some technical results. In this subsection we use some ideas from [4].

Given $u \in W_{A_{t}}^{1, p}(\Omega) \backslash W_{0}^{1, p}(\Omega)$ we consider $v=u \circ \psi_{t}$, so $v \in W_{A}^{1, p}(\Omega) \backslash W_{0}^{1, p}(\Omega)$ and $\nabla v^{T}={ }^{T} \psi_{t}^{\prime} \nabla\left(u \circ \psi_{t}\right)^{T}$, where $\psi_{t}^{\prime}$ denotes the differential matrix of $\psi_{t}$ and ${ }^{T} A$ is the transpose of matrix A. Thus, by the change of variables formula, we have that

$$
\int_{\Omega}|\nabla u|^{p}+|u|^{p} \mathrm{~d} x=\int_{\Omega}\left\{\left.\left.\right|^{T}\left[\psi_{t}^{\prime}\right]^{-1} \nabla v^{T}\right|^{p}+|v|^{p}\right\} J\left(\psi_{t}\right) \mathrm{d} x,
$$

here $J\left(\psi_{t}\right)$ is the usual Jacobian of $\psi_{t}$. Moreover, since $\operatorname{supp}(V) \subset \Omega$, we have that

$$
\int_{\partial \Omega}|u|^{q} \mathrm{~d} S=\int_{\partial \Omega}|v|^{q} \mathrm{~d} S .
$$

In [5] are proved the following asymptotic formulas

$$
\begin{aligned}
{\left[\psi_{t}^{\prime}\right]^{-1}(x) } & =I d-t V^{\prime}(x)+o(t), \\
J\left(\psi_{t}\right)(x) & =1+t \operatorname{div} V(x)+o(t) .
\end{aligned}
$$

Then, by (2.7) and (2.8), we have

$$
\begin{aligned}
\int_{\Omega}|v|^{p} J\left(\psi_{t}\right) \mathrm{d} x & =\int_{\Omega}|v|^{p}\{1+t \operatorname{div} V+o(t)\} \mathrm{d} x \\
& =\int_{\Omega}|v|^{p} \mathrm{~d} x+t \int_{\Omega}|v|^{p} \operatorname{div} V \mathrm{~d} x+o(t)
\end{aligned}
$$


and

$$
\begin{aligned}
\left.\left.\int_{\Omega}\right|^{T}\left[\psi_{t}^{\prime}\right]^{-1} \nabla v^{T}\right|^{p} J\left(\psi_{t}\right) \mathrm{d} x & =\int_{\Omega}\left|\left[I d-t^{T} V^{\prime}+o(t)\right] \nabla v^{T}\right|^{p}\{1+t \operatorname{div} V+o(t)\} \mathrm{d} x \\
& =\int_{\Omega}\left|\nabla v-t^{T} V^{\prime} \nabla v^{T}+o(t)\right|^{p}\{1+t \operatorname{div} V+o(t)\} \mathrm{d} x,
\end{aligned}
$$

since

$$
\left|\nabla v-t^{T} V^{\prime} \nabla v^{T}+o(t)\right|^{p}=|\nabla v|^{p}-p t\left|\nabla v_{t}\right|^{p-2}\left\langle\nabla v,{ }^{T} V^{\prime} \nabla v^{T}\right\rangle+o(t)
$$

we obtain that

$$
\begin{aligned}
\int_{\Omega}\left|{ }^{T}\left[\psi_{t}^{\prime}\right]^{-1} \nabla v^{T}\right|^{p} J\left(\psi_{t}\right) \mathrm{d} x & =\int_{\Omega}|\nabla v|^{p} \mathrm{~d} x+t \int_{\Omega}|\nabla v|^{p} \operatorname{div} V \mathrm{~d} x \\
& -p t \int_{\Omega}|\nabla v|^{p-2}\left\langle\nabla v,{ }^{T} V^{\prime} \nabla v^{T}\right\rangle \mathrm{d} x+o(t) .
\end{aligned}
$$

Thus, we conclude

$$
\begin{aligned}
\int_{\Omega}|\nabla u|^{p}+|u|^{p} \mathrm{~d} x & =\int_{\Omega}\left\{\left.\left.\right|^{T}\left[\psi_{t}^{\prime}\right]^{-1} \nabla v^{T}\right|^{p}+|v|^{p}\right\} J\left(\psi_{t}\right) \mathrm{d} x \\
& =\int_{\Omega}|v|^{p} \mathrm{~d} x+\int_{\Omega}|\nabla v|^{p} \mathrm{~d} x+t \int_{\Omega}\left\{|\nabla v|^{p}+|v|^{p}\right\} \operatorname{div} V \mathrm{~d} x \\
& -p t \int_{\Omega}|\nabla v|^{p-2}\left\langle\nabla v,{ }^{T} V^{\prime} \nabla v^{T}\right\rangle \mathrm{d} x+o(t) .
\end{aligned}
$$

Therefore, we can rewrite (1.5) as

$$
S_{q}(t)=\inf _{v \in W_{A}^{1, p}(\Omega) \backslash W_{0}^{1, p}(\Omega)}\{\rho(v)+t \gamma(v)\}
$$

where

and

$$
\rho(v)=\frac{\int_{\Omega}|\nabla v|^{p}+|v|^{p} \mathrm{~d} x}{\left\{\int_{\partial \Omega}|v|^{q} \mathrm{~d} S\right\}^{p / q}},
$$

$$
\gamma(v)=\frac{\int_{\Omega}\left\{|\nabla v|^{p}+|v|^{p}\right\} \operatorname{div} V \mathrm{~d} x-p \int_{\Omega}|\nabla v|^{p-2}\left\langle\nabla v,{ }^{T} V^{\prime} \nabla v^{T}\right\rangle \mathrm{d} x}{\left\{\int_{\partial \Omega}|v|^{q} \mathrm{~d} S\right\}^{p / q}}+O(t) .
$$

Given $t \geq 0$, let $v_{t} \in W_{A}^{1, p}(\Omega) \backslash W_{0}^{1, p}(\Omega)$ such that $\left\|v_{t}\right\|_{L^{q}(\partial \Omega)}=1$ and

$$
S_{q}(t)=\varphi(t)+t \phi(t)
$$

where

$$
\varphi(t)=\rho\left(v_{t}\right) \text { and } \phi(t)=\gamma\left(v_{t}\right) \quad \forall t \geq 0 .
$$

We observe that $\varphi, \phi: \mathbb{R}_{\geq 0} \rightarrow \mathbb{R}$ and

Lemma 2.1. The function $\phi$ is nonincreasing.

Proof. Let $0 \leq t_{1} \leq t_{2}$. By (2.9), we have that

$$
\begin{aligned}
& \varphi\left(t_{2}\right)+t_{1} \phi\left(t_{2}\right) \geq S_{q}\left(t_{1}\right)=\varphi\left(t_{1}\right)+t_{1} \phi\left(t_{1}\right) \\
& \varphi\left(t_{1}\right)+t_{2} \phi\left(t_{1}\right) \geq S_{q}\left(t_{2}\right)=\varphi\left(t_{2}\right)+t_{2} \phi\left(t_{2}\right) .
\end{aligned}
$$

Subtracting (2.10) from (2.11), we get

$$
\left(t_{2}-t_{1}\right) \phi\left(t_{1}\right) \geq\left(t_{2}-t_{1}\right) \phi\left(t_{2}\right) .
$$


Since $t_{2}-t_{1} \geq 0$, we obtain

$$
\phi\left(t_{1}\right) \geq \phi\left(t_{2}\right) .
$$

This ends the proof.

Remark 2.2. Since $\phi$ is nonincreasing, we have

$$
\phi(t) \leq \phi(0) \quad \forall t \geq 0,
$$

and there exists

$$
\phi\left(0^{+}\right)=\lim _{t \rightarrow 0^{+}} \phi(t) .
$$

Corollary 2.3. The function $\varphi$ is nondecreasing.

Proof. Let $0 \leq t_{1} \leq t_{2}$. Again, by (2.9), we have that

$$
\varphi\left(t_{2}\right)+t_{1} \phi\left(t_{2}\right) \geq S_{q}\left(t_{1}\right)=\varphi\left(t_{1}\right)+t_{1} \phi\left(t_{1}\right)
$$

$\mathrm{SO}$

$$
\varphi\left(t_{2}\right)-\varphi\left(t_{1}\right) \geq t_{1}\left(\phi\left(t_{1}\right)-\phi\left(t_{2}\right)\right) .
$$

Since $0 \leq t_{1} \leq t_{2}$, by Lemma 2.1 , we have that $\phi\left(t_{1}\right)-\phi\left(t_{2}\right) \geq 0$. Then

$$
\varphi\left(t_{2}\right)-\varphi\left(t_{1}\right) \geq 0
$$

that is what we wished to prove.

Now we can prove that $S_{q}(t)$ is continuous at $t=0$.

Theorem 2.4. The function $S_{q}(t)$ is continuous at $t=0$, i.e.,

$$
\lim _{t \rightarrow 0^{+}} S_{q}(t)=S_{q}(0) \text {. }
$$

Proof. Given $t \geq 0$ so, by Corollary 2.3,

$$
S_{q}(t)-S q(0)=\varphi(t)+t \phi(t)-\varphi(0) \geq t \phi(t) .
$$

On the other hand, by (2.9), we have that

$$
S_{q}(t) \leq \varphi(0)+t \phi(0)=S_{q}(0)+t \phi(0) .
$$

Then

$$
t \phi(t) \leq S_{q}(t)-S q(0) \leq t \phi(0) .
$$

Thus, by Remark 2.2,

$$
\lim _{t \rightarrow 0^{+}} S_{q}(t)-S_{q}(0)=0 .
$$

This finishes the proof.

Thus, from Remark 2.2 and Theorem 2.4, we obtain the following corollary:

Corollary 2.5. The function $\varphi$ is continuous at $t=0$, i.e.,

$$
\lim _{t \rightarrow 0^{+}} \varphi(t)=\varphi(0) .
$$

Proof. We observe that

$$
\varphi(t)-\varphi(0)=S_{q}(t)-S_{q}(0)-t \phi(t)
$$

then, by Remark 2.2 and Theorem 2.4,

$$
\lim _{t \rightarrow 0^{+}} \varphi(t)-\varphi(0)=0 .
$$

That proves the result. 
Finally, we prove the following:

Theorem 2.6. The function $\varphi$ is differentiable at $t=0$ and

$$
\frac{\mathrm{d} \varphi}{\mathrm{d} t}(0)=0 .
$$

Proof. Let $0<r<t$. By (2.9), we get

$$
S_{q}(r)=\varphi(r)+r \phi(r) \leq \varphi(t)+r \phi(t),
$$

and

$$
S_{q}(t)=\varphi(t)+t \phi(t) \leq \varphi(r)+t \phi(r) .
$$

So

$$
\frac{r}{t}(\phi(r)-\phi(t)) \leq \frac{\varphi(t)-\varphi(r)}{t} \leq \phi(r)-\phi(t)
$$

hence, taking limits when $r \rightarrow 0^{+}$, by Remark 2.2 and Corollary 2.1, we have that

$$
0 \leq \frac{\varphi(t)-\varphi(0)}{t} \leq \phi\left(0^{+}\right)-\phi(t) .
$$

Now, taking limits when $t \rightarrow 0^{+}$, and again by Remark 2.2 , we get

$$
\lim _{t \rightarrow 0^{+}} \frac{\varphi(t)-\varphi(0)}{t}=0
$$

as we wanted to show.

2.2. Proof of Theorem 1.1. We proceed in three steps.

Step 1. We show that $S_{q}(t)$ is differentiable at $t=0$ and

$$
S_{q}^{\prime}(0)=\phi\left(0^{+}\right) \text {. }
$$

We have that

$$
\frac{S_{q}(t)-S_{q}(0)}{t}=\frac{\varphi(t)-\varphi(0)}{t}-\phi(t)
$$

Then, by Remark 2.2 and Theorem 2.6,

$$
S_{q}^{\prime}(0)=\lim _{t \rightarrow 0^{+}} \frac{S_{q}(t)-S_{q}(0)}{t}=\phi\left(0^{+}\right) .
$$

Step 2. We show that there exists $u$ extremal for $S_{q}(A)$ such that $\|u\|_{L^{q}(\partial \Omega)}=1$ and

$$
\phi\left(0^{+}\right)=\int_{\Omega}\left(|\nabla u|^{p}+|u|^{p}\right) \operatorname{div} V \mathrm{~d} x-p \int_{\Omega}|\nabla u|^{p-2}\left\langle\nabla u,{ }^{T} V^{\prime} \nabla u\right\rangle \mathrm{d} x .
$$

By Theorem 2.1

$$
\left\|v_{t}\right\|_{W^{1, p}(\Omega)}^{p}=\varphi(t) \rightarrow \varphi(0)=S_{q}(0) \text { when } t \rightarrow 0^{+} .
$$

Then there exists $u \in W^{1, p}(\Omega)$ and $t_{n} \rightarrow 0^{+}$when $n \rightarrow \infty$ such that

$$
\begin{aligned}
& v_{t_{n}} \rightarrow u \text { weakly in } W^{1, p}(\Omega), \\
& v_{t_{n}} \rightarrow u \text { strongly in } L^{q}(\partial \Omega), \\
& v_{t_{n}} \rightarrow u \text { a.e. in } \Omega .
\end{aligned}
$$

By (2.15) and (2.16), $u \in W_{A}^{1, p}(\Omega)$ and $\|u\|_{L^{q}(\partial \Omega)}=1$ and by (2.14)

$$
S_{q}(0)=\lim _{n \rightarrow \infty}\left\|v_{t_{n}}\right\|_{W^{1, p}(\Omega)}^{p} \geq\|u\|_{W^{1, p}(\Omega)}^{p} \geq S_{q}(0),
$$


then

$$
S_{q}(0)=\|u\|_{W^{1, p}(\Omega)}^{p} .
$$

Moreover, by (2.13), (2.14) and (2.17), we have that

$$
v_{t_{n}} \rightarrow u \text { strongly in } W^{1, p}(\Omega) \text {. }
$$

Therefore

$$
\begin{aligned}
\phi\left(0^{+}\right) & =\lim _{n \rightarrow \infty} \phi\left(v_{t_{n}}\right) \\
& =\int_{\Omega}\left(|\nabla u|^{p}+|u|^{p}\right) \operatorname{div} V \mathrm{~d} x-p \int_{\Omega}|\nabla u|^{p-2}\left\langle\nabla u,{ }^{T} V^{\prime} \nabla u{ }^{T}\right\rangle \mathrm{d} x .
\end{aligned}
$$

Step 3. Finally, we show that

$$
\begin{aligned}
S_{q}^{\prime}(0) & =\int_{\Omega}\left(|\nabla u|^{p}+|u|^{p}\right) \operatorname{div} V \mathrm{~d} x-p \int_{\Omega}|\nabla u|^{p-2}\left\langle\nabla u,{ }^{T} V^{\prime} \nabla u u^{T}\right\rangle \mathrm{d} x \\
& =-\int_{\partial A}\left|\frac{\partial u}{\partial \nu}\right|^{p}\langle V, \nu\rangle \mathrm{d} S .
\end{aligned}
$$

To show this we require that $u \in C^{2}$. However, this is not true. Since $u$ is an esxtremal for $S_{q}(A)$ and $\|u\|_{L^{q}(\Omega)}=1$, we known that $u$ is weak solution to

$$
\begin{cases}-\Delta_{p} u+|u|^{p-2} u=0 & \text { in } \Omega \backslash \bar{A} \\ |\nabla u|^{p-2} \frac{\partial u}{\partial \nu}=S_{q}(A)|u|^{q-2} u & \text { on } \partial \Omega \\ u=0 & \text { on } \partial A\end{cases}
$$

and by [8] we get that $u$ belongs to the class $C^{1, \delta}$ for some $0<\delta<1$.

In order to overcome this difficulty, we proced as follows. We consider the regularized prblems

$$
\begin{cases}\left.-\operatorname{div}\left(\left|\nabla u^{\varepsilon}\right|^{2}+\varepsilon^{2}\right)^{(p-2) / 2}\right)+\left|u^{\varepsilon}\right|^{p-2} u^{\varepsilon}=0 & \text { in } \Omega \backslash \bar{A} \\ \left.\left|\nabla u^{\varepsilon}\right|^{2}+\varepsilon^{2}\right)^{(p-2) / 2} \frac{\partial u^{\varepsilon}}{\partial \nu}=S_{q}(A)|u|^{q-2} u & \text { on } \partial(\Omega \backslash \bar{A}),\end{cases}
$$

It is well known that the solution $u^{\varepsilon}$ to (2.18) is of class $C^{2, \rho}$ for some $0<\rho<1$ (see $[6])$.

Then, we can perform all of our computations with the functions $u^{\varepsilon}$ and pass to the limit as $\varepsilon \rightarrow 0$ at the end.

We have chosen to work formally with the function $u$ in order to make our arguments more transparent and leave the details to the reader. For a similar approach, see [4].

Since

$$
\begin{aligned}
\operatorname{div}\left(|u|^{p} V\right) & =|u|^{p} \operatorname{div} V+p|u|^{p-2} u\langle\nabla u, V\rangle, \\
\operatorname{div}\left(|\nabla u|^{p} V\right) & =|\nabla u|^{p} \operatorname{div} V+p|\nabla u|^{p-2}\left\langle\nabla u D^{2} u, V\right\rangle,
\end{aligned}
$$

we have that

$$
\begin{aligned}
\int_{\Omega}\left(|\nabla u|^{p}+|u|^{p}\right) \operatorname{div} V \mathrm{~d} x & =\int_{\Omega} \operatorname{div}\left(|u|^{p} V+|\nabla u|^{p} V\right) \mathrm{d} x \\
& -p \int_{\Omega}\left\{|u|^{p-2} u_{0}\langle\nabla u, V\rangle+|\nabla u|^{p-2}\left\langle\nabla u D^{2} u, u V\right\rangle\right\} \mathrm{d} x .
\end{aligned}
$$


Integrating by parts, we obtain

$$
\begin{aligned}
\int_{\Omega} \operatorname{div}\left(|u|^{p} V+|\nabla u|^{p} V\right) \mathrm{d} x & =\int_{\partial \Omega}\left(|u|^{p}+|\nabla u|^{p}\right)\langle V, \nu\rangle \mathrm{d} S-\int_{\partial A}\left(|u|^{p}+|\nabla u|^{p}\right)\langle V, \nu\rangle \mathrm{d} S \\
& =-\int_{\partial A}|\nabla u|^{p}\langle V, \nu\rangle \mathrm{d} S .
\end{aligned}
$$

where the las equality follows from the fact that $\operatorname{supp}(V) \subset \Omega$ and $u=0$ on $\partial A$.

Thus

$$
\begin{aligned}
S_{q}^{\prime}(0) & =-\int_{\partial A}|\nabla u|^{p}\langle V, \nu\rangle \mathrm{d} S-p \int_{\Omega}|u|^{p-2} u\left\langle\nabla u_{0}, V\right\rangle \mathrm{d} x \\
& -p \int_{\Omega}|\nabla u|^{p-2}\left\langle\nabla u,{ }^{T} V^{\prime} \nabla u+{ }^{T} D^{2} u V^{T}\right\rangle \mathrm{d} x \\
& =-\int_{\partial A}|\nabla u|^{p}\langle V, \nu\rangle \mathrm{d} S-p \int_{\Omega}\left|u_{0}\right|^{p-2} u\langle\nabla u, V\rangle \mathrm{d} x \\
& -p \int_{\Omega}|\nabla u|^{p-2}\langle\nabla u, \nabla(\langle\nabla u, V\rangle)\rangle \mathrm{d} x .
\end{aligned}
$$

Since $u$ is a week solution of (1.3) as $\lambda=S_{q}(0)$ and $\operatorname{supp}(V) \subset \Omega$ we have

$$
S_{q}^{\prime}(0)=-\int_{\partial A}|\nabla u|^{p}\langle V, \nu\rangle \mathrm{d} S
$$

Then, noticing that $\nabla u=\frac{\partial u}{\partial \nu} \nu$, the proof is complete.

\section{LaCK of Symmetry in the Ball}

In this section we consider the case where $\Omega=B_{R}$ and $A=B_{r}$ with $r<R$ and show Theorem 1.3, Theorem 1.4 and Proposition 1.5. The proofs are based on the argument of [2] and [7] adapted to our problem. In order to simplify notations, we write $S_{q}(r)$ instead $S_{q}\left(B_{r}\right)$.

First we proof Theorem 1.3, for this we need the following proposition

Proposition 3.1. Let $1<q<p$. The nonnegative solution of (1.3) is unique.

Proof. Suppose that there exist two nonnegative solutions $u$ and $v$ of (1.3). By Remark 1.2 it follows that $u, v>0$ on $\partial \Omega$. Let $v_{n}=v+\frac{1}{n}$ with $n \in \mathbb{N}$, using first Piccone's identity (see [1]) and the weak formulation of (1.3) we have

$$
\begin{aligned}
0 & \leq \int_{B_{R}}|\nabla u|^{p} \mathrm{~d} x-\int_{B_{R}}\left|\nabla v_{n}\right|^{p-2} \nabla v_{n} \nabla\left(\frac{u^{p}}{v_{n}^{p-1}}\right) \mathrm{d} x \\
& =\int_{B_{R}}|\nabla u|^{p} \mathrm{~d} x-\int_{B_{R}}|\nabla v|^{p-2} \nabla v \nabla\left(\frac{u^{p}}{v_{n}^{p-1}}\right) \mathrm{d} x \\
& =-\int_{B_{R}} u^{p} \mathrm{~d} x+\lambda \int_{\partial B_{R}} u^{q} \mathrm{~d} S+\int_{B_{R}} v^{p-1} \frac{u^{p}}{v_{n}^{p-1}} \mathrm{~d} x-\lambda \int_{\partial B_{R}} v^{q-1} \frac{u^{p-1}}{v_{n}^{p-1}} \mathrm{~d} S \\
& \leq \lambda \int_{\partial B_{R}} u^{q} \mathrm{~d} S-\lambda \int_{\partial B_{R}} v^{q-1} \frac{u^{p-1}}{v_{n}^{p-1}} \mathrm{~d} S .
\end{aligned}
$$


Thus, by the Monotone Convergence Theorem,

$$
\begin{aligned}
0 & \leq \int_{\partial B_{R}} u^{q} \mathrm{~d} S-\int_{\partial B_{R}} v^{q-1} \frac{u^{p-1}}{v^{p-1}} \mathrm{~d} S \\
& =\int_{\partial B_{R}} u^{q}\left(u^{q-1}-v^{q-1}\right) \mathrm{d} S .
\end{aligned}
$$

Note that the role of $u$ and $v$ in the above equation are exchangeable. Therefore, subtracting we get

$$
0 \leq \int_{\partial B_{R}}\left(u^{q}-v^{q}\right)\left(u^{q-1}-v^{q-1}\right) \mathrm{d} S .
$$

Since $q<p$ we have that $u \equiv v$ on $\partial B_{R}$. Then, by uniqueness of solution to the Dirichlet problem, we get $u \equiv v$ in $B_{R}$.

Remark 3.2. As the problem (1.3) is rotationally invariant, by uniqueness we obtain that the nonnegative solution of (1.3) must be radial. Therefore, if $\Omega=B_{R}$, $A=B_{r}$ and $1<q \leq p$ we can suppose that the extremal for $S_{q}(r)$ found in the Theorem 1.1 is nonnegative and radial.

Now we can prove the Theorem 1.3 ,

Proof of Theorem 1.3. We consider $\Omega=B_{R}, A=B_{r}$ and $1<q \leq p$. By Theorem 1.3 and Remark 3.2 there exist a nonnegative and radial normalized extremal for $S_{q}(r)$ such that

Since $u$ is radial

$$
S_{q}^{\prime}(0)=-\int_{\partial B_{r}}\left|\frac{\partial u}{\partial \nu}\right|^{p}\langle V, \nu\rangle \mathrm{d} S
$$

where $c$ is a constant.

$$
\frac{\partial u}{\partial \nu} \equiv c \text { on } \partial B_{r}
$$

Thus, using that we are dealing with deformations $V$ that preserves the volume of the $B_{r}$, we have that

$$
S_{q}^{\prime}(0)=-c^{p} \int_{\partial B_{r}}\langle V, \nu\rangle \mathrm{d} S=c^{p} \int_{B_{r}} \operatorname{div}(V) \mathrm{d} x=0 .
$$

To prove Theorem 1.4, we need two previous results.

Proposition 3.3. Let $r>0$ fixed. Then, there exists a positive radial function $u_{0}$ such that

$$
\begin{cases}-\Delta_{p} u+|u|^{p-2} u=0 & \text { in } \mathbb{R}^{N} \backslash B_{r}, \\ u=0 & \text { on } \partial B_{r} .\end{cases}
$$

This $u_{0}$ is unique up to a constant factor and for any $R>r$ the restriction of $u_{0}$ to $B_{R}$ is the first eigenfunction of (1.3) with $q=p$.

Proof. For $R>r$, let $u_{R}$ be the unique solution of the Dirichlet problem

$$
\left\{\begin{array}{l}
\Delta_{p} u_{R}=\left|u_{R}\right|^{p-2} u_{R} \quad \text { in } B_{R} \backslash \overline{B_{r}} \\
u(R)=1 \\
u(r)=0 .
\end{array}\right.
$$


Then, by uniqueness, $u_{R}$ is a nonnegative and radial function. Moreover, by the regularity theory and maximum principle we have $\frac{\partial u_{R}}{\partial \nu}(r) \neq 0$ (see $[8,11]$ ). Thus, for any $R>r$, we define the restriction of $u_{0}$ by

$$
u_{0}=\frac{u_{R}}{\frac{\partial u_{R}}{\partial \nu}(r)} .
$$

By uniqueness of the Dirichlet problem, it is easy to check that $u_{0}$ is well defined and is a nonnegative radial solution of (3.19). Furthermore, by the simplicity of $S_{p}(r), u_{0}$ is the eigenfunction associated to $S_{p}(r)$ for every $R>r$.

Proposition 3.4. Let $v$ be a radial solution of (1.3). Then $v$ is a multiple of $u_{0}$. In particular any radial minimizer of (1.2) is a multiple of $u_{0}$.

Proof. Let $a>0$ be such that $v=a u_{0}$ on $\partial B(0, R)$. Then $v$ and $a u_{0}$ are two solutions to the Dirichlet problem $\Delta_{p} w=w^{p-1}$ and $w=v$ on $\partial\left(B_{R} \backslash \overline{B_{r}}\right)$. Hence, by uniqueness, we have that $v=a u_{0}$ in $B_{R}$.

Remark 3.5. If $1<q<p$ then the solution of (1.3), by Remark 3.2 and Proposition 3, is a multiple of $u_{0}$.

Now we can deal with the proof of Theorem 1.4.

Proof of Theorem 1.4. Let $R>r$ be fixed and consider $u_{0}$ to be the nonnegative radial function given by Proposition 3.3 such that that $u_{0}=1$ on $\partial B_{R}$. Then, by Proposition 3.4 , it is enough to prove that $u_{0}$ is not a minimizer for $S_{q}(r)$ when $q>Q(R)$.

First let us move this symmetric configuration in the $x_{1}$ direction. For any $t \in \mathbb{R}$ and $x \in \mathbb{R}^{N}$ we denote $x_{t}=\left(x_{1}-t, x_{2}, \ldots, x_{N}\right)$ and define

$$
U(t)(x)=u_{0}\left(x_{t}\right)
$$

Observe that $U$ vanishes in $A_{t}:=B_{r}\left(t e_{1}\right)$ (the ball with center $t e_{1}$ and radius $r$ ) a subset of $B_{R}$ of the same measure of $B_{r}$ for all $t$ small.

Consider the function

$$
h(t)=\frac{f(t)}{g(t)}
$$

where

$$
f(t)=\int_{B_{R}}|\nabla U|^{p}+U^{p} \mathrm{~d} x \text { and } g(t)=\left(\int_{\partial B_{R}} U^{q} \mathrm{~d} S\right)^{\frac{p}{q}} .
$$

We observe that $h(0)=0$ and since $h$ is an even function, we have $h^{\prime}(0)=0$. Now,

$$
h^{\prime \prime}(0)=\left.\frac{f^{\prime \prime} g^{2}-f g g^{\prime \prime}-2 f^{\prime} g g^{\prime}-2 f g g^{\prime}}{g^{3}}\right|_{t=0} .
$$

Next we compute these terms. First, since $u_{0}$ is the first eigenfunction of (1.3) with $q=p$ and $u_{0}=1$ on $\partial B_{R}$ we get

$$
f(0)=S_{p}(r)\left|\partial B_{R}\right| \text { and } g(0)=\left|\partial B_{R}\right|^{\frac{p}{q}} .
$$

Thus, by Gauss-Green's Theorem and using the fact that $u_{0}$ is radial, we get

$$
f^{\prime}(0)=-\int_{B_{R}} \frac{\partial}{\partial x_{1}}\left(\left|\nabla u_{0}\right|^{p}+u_{0}^{p}\right) \mathrm{d} x=\int_{\partial B_{R}}\left(\left|\nabla u_{0}\right|^{p}+u_{0}^{p}\right) \nu_{1} \mathrm{~d} S=0 .
$$


Again, since $u_{0}$ is radial,

$$
g^{\prime}(0)=\frac{p}{q}\left(\int_{\partial B_{R}} u^{q} \mathrm{~d} S\right)^{\frac{p}{q}-1}\left(\int_{\partial B_{R}} \frac{\partial u^{q}}{\partial x_{1}} \mathrm{~d} S\right)=0 .
$$

Finally, using that $u_{0}=1$ on $\partial B_{R}$, we obtain

$$
g^{\prime \prime}(0)=p\left|\partial B_{R}\right|^{\frac{p}{q}-1} \int_{\partial B_{R}}(q-1)\left(\frac{\partial u_{0}}{\partial x_{1}}\right)^{2}+\frac{\partial^{2} u_{0}}{\partial x_{1}^{2}} \mathrm{~d} S
$$

and, by the Gauss-Green's Theorem

$$
\begin{aligned}
f^{\prime \prime}(0) & =p \int_{B_{R}} \frac{\partial}{\partial x_{1}}\left(\frac{1}{2}\left|\nabla u_{0}\right|^{p-2} \frac{\partial\left|\nabla u_{0}\right|^{2}}{\partial x_{1}}+\frac{1}{p} \frac{\partial u_{0}^{p}}{\partial x_{1}}\right) \mathrm{d} x \\
& =p \int_{\partial B_{R}}\left(\frac{1}{2}\left|\nabla u_{0}\right|^{p-2} \frac{\partial\left|\nabla u_{0}\right|^{2}}{\partial x_{1}}+\frac{1}{p} \frac{\partial u_{0}^{p}}{\partial x_{1}}\right) \nu_{1} \mathrm{~d} S .
\end{aligned}
$$

Then

$$
\begin{aligned}
h^{\prime \prime}(0)= & \frac{p}{\left|\partial B_{R}(0)\right|^{p / q}}\left[\int_{\partial B_{R}}\left(\frac{1}{2}\left|\nabla u_{0}\right|^{p-2} \frac{\partial\left|\nabla u_{0}\right|^{2}}{\partial x_{1}}+\frac{1}{p} \frac{\partial u_{0}^{p}}{\partial x_{1}}\right) \nu_{1} \mathrm{~d} S\right. \\
& \left.-S_{p}(r) \int_{\partial B_{R}}(q-1)\left(\frac{\partial u_{0}}{\partial x_{1}}\right)^{2}+\frac{\partial^{2} u_{0}}{\partial x_{1}^{2}} \mathrm{~d} S\right] .
\end{aligned}
$$

Thus, since $u_{0}$ is radial, we get

$$
\begin{aligned}
h^{\prime \prime}(0)= & \frac{p}{N\left|\partial B_{R}(0)\right|^{p / q}}\left[\int_{\partial B_{R}}\left(\frac{1}{2}\left|\nabla u_{0}\right|^{p-2} \frac{\partial\left|\nabla u_{0}\right|^{2}}{\partial \nu}+\frac{1}{p} \frac{\partial u_{0}^{p}}{\partial \nu}\right) \mathrm{d} S\right. \\
& \left.-S_{p}(r) \int_{\partial B_{R}}(q-1)\left|\nabla u_{0}\right|^{2}+\Delta u_{0} \mathrm{~d} S\right] .
\end{aligned}
$$

Now, by definition, $u_{0}(x)=u_{0}(|x|)$ and $\alpha$ satisfies

$$
\left(s^{N-1}\left|u_{0}^{\prime}\right|^{p-1} u_{0}^{\prime}\right)^{\prime}=s^{N-1} u_{0}^{p-1} \quad \forall s>r
$$

with $u_{0}(R)=0$ and $u_{0}(r)=0$, moreover, by Proposition 3.3, we have

$$
u_{0}^{\prime}(s)^{p-1}=S_{p}(r) u_{0}(s)^{p-1} \quad \forall s>r .
$$

Then

$$
\frac{1}{2}\left|\nabla u_{0}\right|^{p-2} \frac{\partial\left|\nabla u_{0}\right|^{2}}{\partial \nu}+\frac{1}{p} \frac{\partial u_{0}^{p}}{\partial \nu}=\frac{S_{p}(r)^{\frac{1}{p-1}}}{p-1}\left(1-\frac{N-1}{R} S_{p}(r)\right)+S_{p}(r)^{\frac{1}{p-1}}
$$

and

$$
\begin{aligned}
S_{p}(r)\left[(q-1)\left|\nabla u_{0}\right|^{2}+\Delta u_{0}\right] & =(q-1) S_{p}(r)^{\frac{p+1}{p-1}}+\frac{S_{p}(r)^{\frac{1}{p-1}}}{p-1}\left(1-\frac{N-1}{R} S_{p}(r)\right) \\
& +\frac{N-1}{R} S_{p}(r)^{\frac{p}{p-1}} .
\end{aligned}
$$

Therefore

$$
h^{\prime \prime}(0)=\frac{p S_{p}^{\frac{1}{p-1}}}{N\left|\partial B_{R}\right|^{\frac{p}{q}-1}}\left[1-(q-1) S_{p}(r)^{\frac{p}{p-1}}-\frac{N-1}{R} S_{p}(r)\right] .
$$


Thus, if $q>Q(R)$ we get that $h^{\prime \prime}(0)<0$ and so 0 is a strict local maxima of $\psi$. So we have proved that

$$
S_{q}(r)=h(0)>h(t) \geq S_{q}\left(B_{r}\left(t e_{1}\right)\right)
$$

for all t small. Therefore a symmetric configuration is not optimal.

To finish the paper we prove Proposition 1.5.

Proof of Proposition 1.5. We proceed in two step.

Step 1. First we show that, for $R>r, S_{p}(R, r)=S_{p}(r)$ verifies the differential equation

$$
\frac{\partial S_{p}}{\partial R}=-\frac{N-1}{R} S_{p}+1-(p-1) S_{p}^{\frac{p}{p-1}}
$$

with the condition

$$
\left.S_{p}\right|_{R=r}=+\infty .
$$

Again we consider $u_{0}(x)=u_{0}(|x|)$ the nonnegative radial function given by Proposition 3.3. Thus, for all $R>r$, we get

$$
\left\{\begin{array}{l}
(p-1)\left(u_{0}^{\prime}\right)^{p-2} u_{0}^{\prime \prime}+\frac{N-1}{R}\left(u_{0}^{\prime}\right)^{p-1}=u_{0}^{p-1} \\
u_{0}^{\prime}(R)^{p-1}=S_{p} u_{0}(R)^{p-1} \\
u_{0}(r)=0
\end{array}\right.
$$

Then

$$
S_{p}=\left(\frac{u_{0}^{\prime}(R)}{u_{0}(R)}\right)^{p-1}
$$

Thus

$$
\begin{aligned}
\frac{\partial S_{p}}{\partial R} & =(p-1)\left(\frac{u_{0}^{\prime}(R)}{u_{0}(R)}\right)^{p-2} \frac{u_{0}^{\prime \prime}(R) u_{0}(R)-u_{0}^{\prime}(R)^{2}}{u_{0}(R)^{2}} \\
& =(p-1)\left(\frac{u_{0}^{\prime}(R)}{u_{0}(R)}\right)^{p-2} \frac{u_{0}^{\prime \prime}(R)}{u_{0}(R)}-(p-1) S_{p}^{\frac{p}{p-1}} \\
& =(p-1) \frac{u_{0}^{\prime}(R)^{p-2} u_{0}^{\prime \prime}(R)}{u_{0}(R)^{p-1}}-(p-1) S_{p}^{\frac{p}{p-1}} \\
& =1-\frac{N-1}{R} S_{p}-(p-1) S_{p}^{\frac{p}{p-1}} .
\end{aligned}
$$
Then

On the other hand, since (by definition) $\frac{\partial u_{0}}{\partial \nu} \equiv 1$ on $\partial B_{r}$, we get that $u^{\prime}(r)=1$.

$$
\lim _{R \rightarrow r} S_{p}=\lim _{R \rightarrow r}\left(\frac{u_{0}^{\prime}(R)}{u_{0}(R)}\right)^{p-1}=+\infty .
$$

Now, it is easy to check that $\lim _{R \rightarrow r} Q(R)=1^{-}$.

Step 2. Finally, we prove that

$$
\lim _{R \rightarrow+\infty} Q(R)=p .
$$

We begin differentiating (3.20) to obtain

$$
\frac{\partial^{2} S_{p}}{\partial R^{2}}=\frac{N-1}{R^{2}} S_{p}-\frac{N-1}{R} \frac{\partial S_{p}}{\partial R}-p S_{p}^{\frac{1}{p-1}} \frac{\partial S_{p}}{\partial R} .
$$


Then, since $S_{p}>0$, at any critical point $\left(S_{p}^{\prime}=0\right)$ we have that $S_{p}^{\prime \prime}>0$. Thus, $S_{p}$ has at most one critical point, which is a minimum. If $S_{p}$ has a minimum, then there exist $R_{0}>r$ such that $S_{p}^{\prime}\left(R_{0}\right)=0$. Moreover, since $S_{p}^{\prime}(R) \neq 0$ for any $R \neq R_{0}$ and $S_{p} \rightarrow+\infty$ as $R \rightarrow r$ and by (3.20), we get that $S_{p}^{\prime}<0$ for all $r<R<R_{0}$ and $S_{p}^{\prime}>0$ for all $R>R_{0}$. Thus, using again (3.20) we have that $S_{p}^{\frac{p}{p-1}}<\frac{1}{p-1}$ for all $R>R_{0}$. Then $S_{p}$ is strictly increasing as a function of $R$ and bonded for all $R>R_{0}$. Consequently $S_{p}^{\prime} \rightarrow 0$ as $R \rightarrow+\infty$. It follows, by (3.20), that $S_{p}^{\frac{p}{p-1}} \rightarrow \frac{1}{p-1}$ as $R \rightarrow+\infty$. On the other hand using (1.6) and (3.20) we see that

$$
S_{p}=(Q(R)-p) S_{p}^{\frac{p}{p-1}} .
$$

So, if $S_{p}$ has a minimum, we get that $Q(R)>p$ for all $R>R_{0}$ and $Q(R) \rightarrow p^{+}$as $R \rightarrow+\infty$. Now, If $S_{p}$ has not critical points so $S_{p}^{\prime} \neq 0$ for all $R>r$ and using that $S_{p} \rightarrow+\infty$ as $R \rightarrow r$ and (3.20) we get that $S_{p}^{\prime}<0$ for all $R>r$. Consequently, in this case, $S_{p}$ is strictly decreasing and therefore $S_{p}^{\prime} \rightarrow 0$ as $R \rightarrow+\infty$ and by (3.20) we have that $S_{p} \rightarrow \frac{1}{p-1}$ as $R \rightarrow+\infty$. Then, if $S_{p}$ has not critical points, we get $Q(R)<p$ and $Q(R) \rightarrow p^{-}$as $R \rightarrow+\infty$.

Acknowledgements I want to thank J. Fernández Bender for his throughout reading of the manuscript that help us to improve the presentation of paper.

\section{REFERENCES}

1. Walter Allegretto and Yin Xi Huang, A Picone's identity for the p-Laplacian and applications, Nonlinear Anal. 32 (1998), no. 7, 819-830.

2. Julián Fernández Bonder, Pablo Groisman, and Julio D. Rossi, Optimization of the first Steklov eigenvalue in domains with holes: a shape derivative approach, Ann. Mat. Pura Appl. (4) 186 (2007), no. 2, 341-358.

3. Julián Fernández Bonder and Julio D. Rossi, A nonlinear eigenvalue problem with indefinite weights related to the Sobolev trace embedding, Publ. Mat. 46 (2002), no. 1, 221-235.

4. Jorge García Melián and José Sabina de Lis, On the perturbation of eigenvalues for the $p$ Laplacian, C. R. Acad. Sci. Paris Sér. I Math. 332 (2001), no. 10, 893-898.

5. A. Henrot and M. Pierre, Optimization de forme: un analyse géométric. mathematics and applications, vol. 48, Springer-Verlag, 2005.

6. O. A. Ladyženskaja, V. A. Solonnikov, and N. N. Ural'ceva, Linear and quasilinear equations of parabolic type, Translated from the Russian by S. Smith. Translations of Mathematical Monographs, Vol. 23, American Mathematical Society, Providence, R.I., 1967.

7. Enrique J. Lami Dozo and Olaf Torné, Symmetry and symmetry breaking for minimizers in the trace inequality, Commun. Contemp. Math. 7 (2005), no. 6, 727-746.

8. Gary M. Lieberman, Boundary regularity for solutions of degenerate elliptic equations, Nonlinear Anal. 12 (1988), no. 11, 1203-1219.

9. Sandra Martínez and Julio D. Rossi, Isolation and simplicity for the first eigenvalue of the p-Laplacian with a nonlinear boundary condition, Abstr. Appl. Anal. 7 (2002), no. 5, 287-293.

10. M. W. Steklov, Sur les problémes fondamentaux en physique mathématique, Ann. Sci. Ecole Norm. Sup. 19 (1902), 445-490.

11. J. L. Vázquez, A strong maximum principle for some quasilinear elliptic equations, Appl. Math. Optim. 12 (1984), no. 3, 191-202.

Leandro M. Del Pezzo

Departamento de Matemática, FCEyn, Universidad de Buenos Aires,

Pabellón I, Ciudad Universitaria (1428), Buenos Aires, Argentina.

E-mail address: 1dpezzo@dm.uba.ar 